\title{
Modelling of ensuring slope stability
}

\author{
Slávka Harabinová ${ }^{1, *}$ and Eva Panulinová ${ }^{1}$ \\ ${ }^{1}$ Technical University of Kosice, Faculty of Civil Engineering, Vysokoškolská 4, 04200 Kosice, \\ Slovak Republic
}

\begin{abstract}
Analysis and assessment of the slopes stability are an important in geotechnical engineering for all the times. The first and foremost requirement for the modelling and design of slope is to guarantee their safety and reliability during their service life. In analysing the overall stability of the ground, of soil or rock, all relevant modes of failure shall be taken into account. When modelling a slope stability processes, it should be considered: soil layering, occurrence and inclination of discontinuities, seepage and pore-water pressure distribution, short- and long-term stability, type of failure (circular or non-circular surface; toppling; flow), using of numerical methods. The paper deals with the modelling of ensuring slope stability.
\end{abstract}

\section{Introduction}

The issue of slope stability is highly topical not only in assessing the stability of existing slopes, but also in the realization of excavations in the construction of various types of industrial or engineering buildings [1]. During excavation work of natural slopes, the slope face may deform and this can lead to slope failure. The movement may continue if no remedial work was implemented on the cut slope [2].

Slovakia is a relatively hilly country, and so especially in the construction of civil engineering (highway roads, railways) it is necessary to realize not only high embankments, but also cuts. For ensuring stability of embankments or notches, the engineering-geological conditions of the site must be known.

Assessment of the slope stability at notch and design of the notch stability structures are carried out during each phase of the realization [3-5].

Methodology for assessing the safety of structures according to the degree of safety is historically the oldest and still widespread. Its main advantage is simplicity and clarity. The safety of the structure is demonstrated by the factor of safety

$$
F_{\mathrm{s}}=X_{\text {pas }} / X_{a k t}
$$

Where $F_{s}$ is calculated factor of safety, $X_{\text {pas }}$ is passive forces, i.e. the forces resisting movement (thus ensuring the slope stability), $X_{a k t}$ is active forces, i.e. the forces to those driving movement (thus threatening the slope stability).

\footnotetext{
*Corresponding author: slavka.harabinova@tuke.sk
} 
Reduction coefficients be used in the assessing slope stability according to the limit states method.

If the soil properties values $(c, \varphi, \gamma)$ are adjusted by a reduction coefficients $\left(\gamma_{m}\right)$ in the calculation and assessment of slope stability, it is sufficient to prove a stability greater than $1\left(F_{\mathrm{s}}>1.0\right)$. If characteristic values in the calculation are used, the reliability shall be greater than $1.5\left(F_{\mathrm{s}}>1.5\right)$. Designing optimal and safety slopes, it is important to determine the geometry of slope, type of rock/soil and their characteristics.

\section{Description of the construction and Engineering-geological conditions of locality}

We analysed the ensuring of slope stability to be realized in the north of Slovakia. We made the slope stability model to protect the deep cutting. Engineering-geological conditions were determined by geological survey. They are represented by Quaternary sediments deluvial clays and proluvial-deluvial clays dominate the area. Deluvial clays are represented mainly by clays of medium plasticity - CI, group F6. Proluvial-deluvial clays are represented mainly by clays of medium plasticity of consistent-soft consistence - CI,t group F6.

Bedrock of Quaternary sediments is made by Paleogene silty-clayey complex. Completely weathered siltstones and claystones occur have the character of eluvial medium plasticity bedrock of consistent consistence (CL-F6). Weathered siltstones and claystones have the character of interbeded soil of medium plasticity of consistent and hard consistence. They represent the change from consistent soil F6 to weathered half-rocks R6. Slightly weathered and healthy siltstones and claystones have the character of more consistent half-rocks R4-R5. Ground water level has not been found. The results and arrangement of the layers are shown in the Table 1.

Table 1. Soil geotechnical parameters used for modelling.

\begin{tabular}{|l|c|c|c|c|}
\hline \multicolumn{1}{|c|}{ Characteristic of rock/soil } & $\gamma\left(\mathbf{k N m}^{-\mathbf{3}}\right)$ & $\left.\boldsymbol{\varphi}_{\text {ef }} \mathbf{(}^{\mathbf{0}}\right)$ & $\boldsymbol{c}_{\boldsymbol{e f}}(\mathbf{k P a})$ & $\boldsymbol{v}$ \\
\hline Deluvial clays (F6 - CI,t) & 20.5 & 24.0 & 10.0 & 0.4 \\
\hline $\begin{array}{l}\text { Completely weathered siltstones and } \\
\text { claystones R6, have the character (F6 - CL,p) }\end{array}$ & 21.0 & 24.0 & 12.0 & 0.4 \\
\hline $\begin{array}{l}\text { Weathered siltstones and claystones R6, have } \\
\text { the character (F6 - CL,p) }\end{array}$ & 21.5 & 26.0 & 20.0 & 0.35 \\
\hline $\begin{array}{l}\text { Slightly weathered and healthy siltstones and } \\
\text { claystones (R4) }\end{array}$ & 24.0 & 35.0 & 15.0 & 0.3 \\
\hline
\end{tabular}

\section{Alternative solutions to ensure slope stability of deep cutting}

Due to big height of the proposed notch (maximum height is $13.6 \mathrm{~m}$ ) and due to various geological conditions have been proposed several variant to ensure the slope stability. Calculation is provided in four variants (Variant 1 to 4 ):

- Variant I - Cut wall is proposed in the gradient 5:1.

- Variant II - Cut wall is proposed in the gradient 5:1 horizontally divided by the one slope bench with the width $1.0 \mathrm{~m}$.

- Variant III - Cut wall is proposed in the gradient 5:1 horizontally divided by the two slope benches with the width $1.0 \mathrm{~m}$.

- Variant IV - Cut wall is proposed in the gradient 1:1.25. 
Slope stability of notches is determined on generated critical sliding surfaces, which were found by using of Bishop, Petterson and Sarma, Spencer method after slope cutting. Calculation and assessment of slope stability was carried out by using program "Slope stability", which is a sub-program of GEO 5 by company FINE Ltd [6].

A factor of safety is defined as the ratio of the forces resisting movement to those driving movement, i.e. the ratio between the passive and active forces. In general, if the factor of safety of a slope is within the interval between 0 and 1.0, the slope is unstable. The value over 1.0 indicates that the slope is considered stable.

Typical mechanical soil properties are with Engineering-geological survey in accordance. The Fig. 1 shows the cut wall proposed in the Variant I to IV. The calculated factors of safety for the proposed variants are shown in the Table 2. The calculated factors of safety for the proposed variants (Variant III and Variant IV) are shown in the table below.

Terrain shape and arrangement of layers
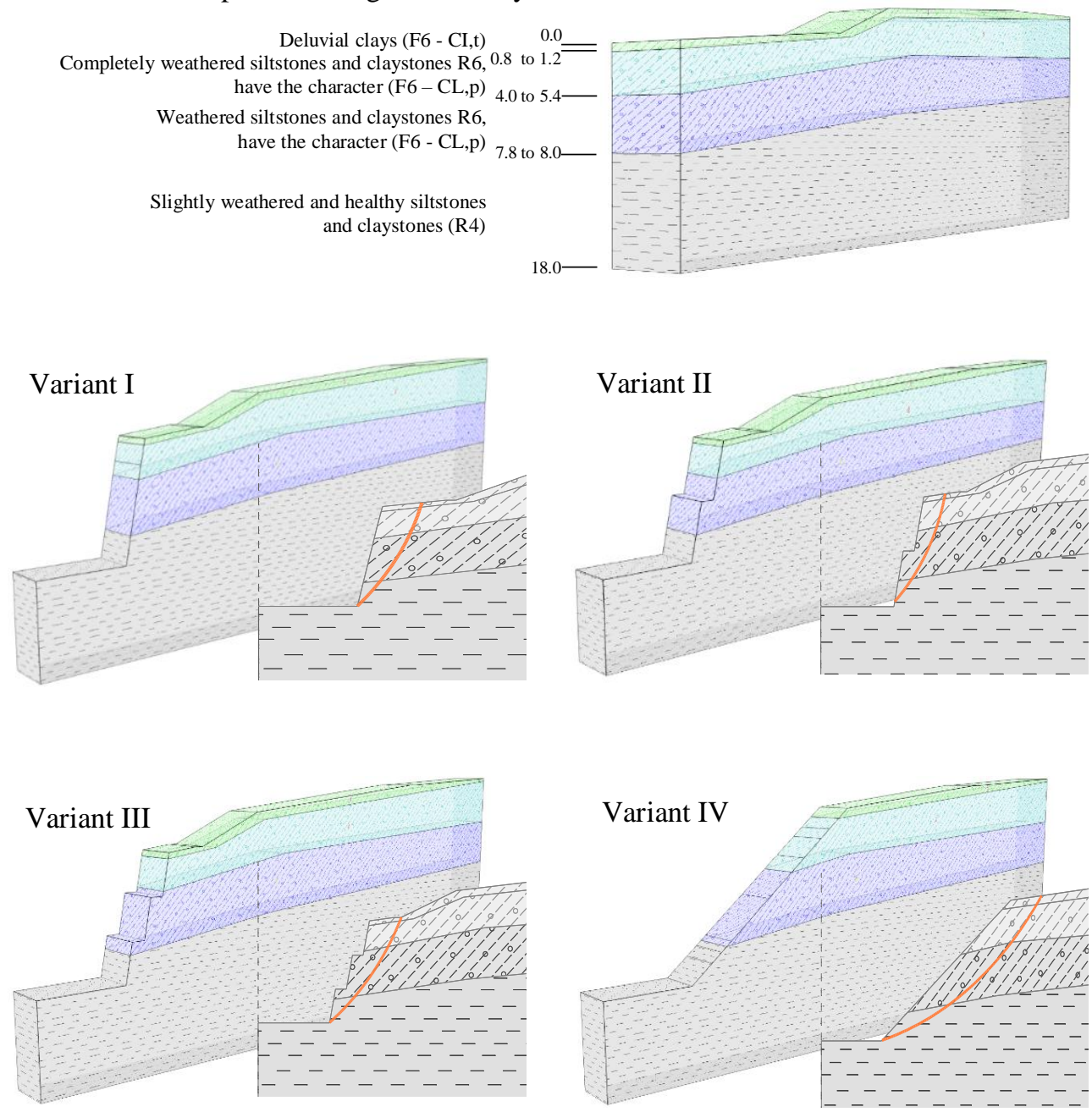

Fig. 1. Cut wall is designed in Variants I to IV and the critical sliding surfaces indicated by red line were found using the Bishop method. 
First variant - Cut wall was proposed in the gradient 5:1. Because the calculated factors of safety for the proposed variant are not satisfactory (Table 2), Variant II in the gradient 5:1 horizontally divided by one slope bench with the width $1.0 \mathrm{~m}$ was proposed. The calculated factors of safety for the proposed variant II are also unsatisfactory, so Variant III in the gradient 5:1 horizontally divided by two slope bench with the width $1.0 \mathrm{~m}$ was proposed. Variant IV - Cut wall is proposed in the gradient 1:1.25, because the calculated factors of safety for the proposed Variant III are also unsatisfactory (Table 2).

Table 2. Factors of safety calculated for Variant I to Variant IV.

\begin{tabular}{|c|c|c|c|c|}
\hline \multirow{2}{*}{ Methods } & \multicolumn{4}{|c|}{ Factor of safety $F s$} \\
\cline { 2 - 5 } & Variant I & Variant II & Variant III & Variant IV \\
\hline Bishop & 0.72 & 0.79 & 0.87 & 1.31 \\
\hline Petterson & 0.72 & 0.79 & 0.87 & 1.29 \\
\hline Spencer & 0.77 & 0.81 & 0.91 & 1.4 \\
\hline Sarma & 0.76 & 0.83 & 0.93 & 1.36 \\
\hline
\end{tabular}

Variant IV is satisfactory, but it occupies a large area and that means a large volume of earthwork.

\section{Discussion}

Based on the calculations and results shown in Fig. 2, the Variant I to III did not satisfy the assessment of slope stability. The calculated safety factors, the point that Variant I to III are an increased risk of landslide. Variant IV in which Wall cutting was proposed in the gradient 1:1.25 satisfies the assessment of slope stability. It has the greatest safety factors for all used calculation methods.

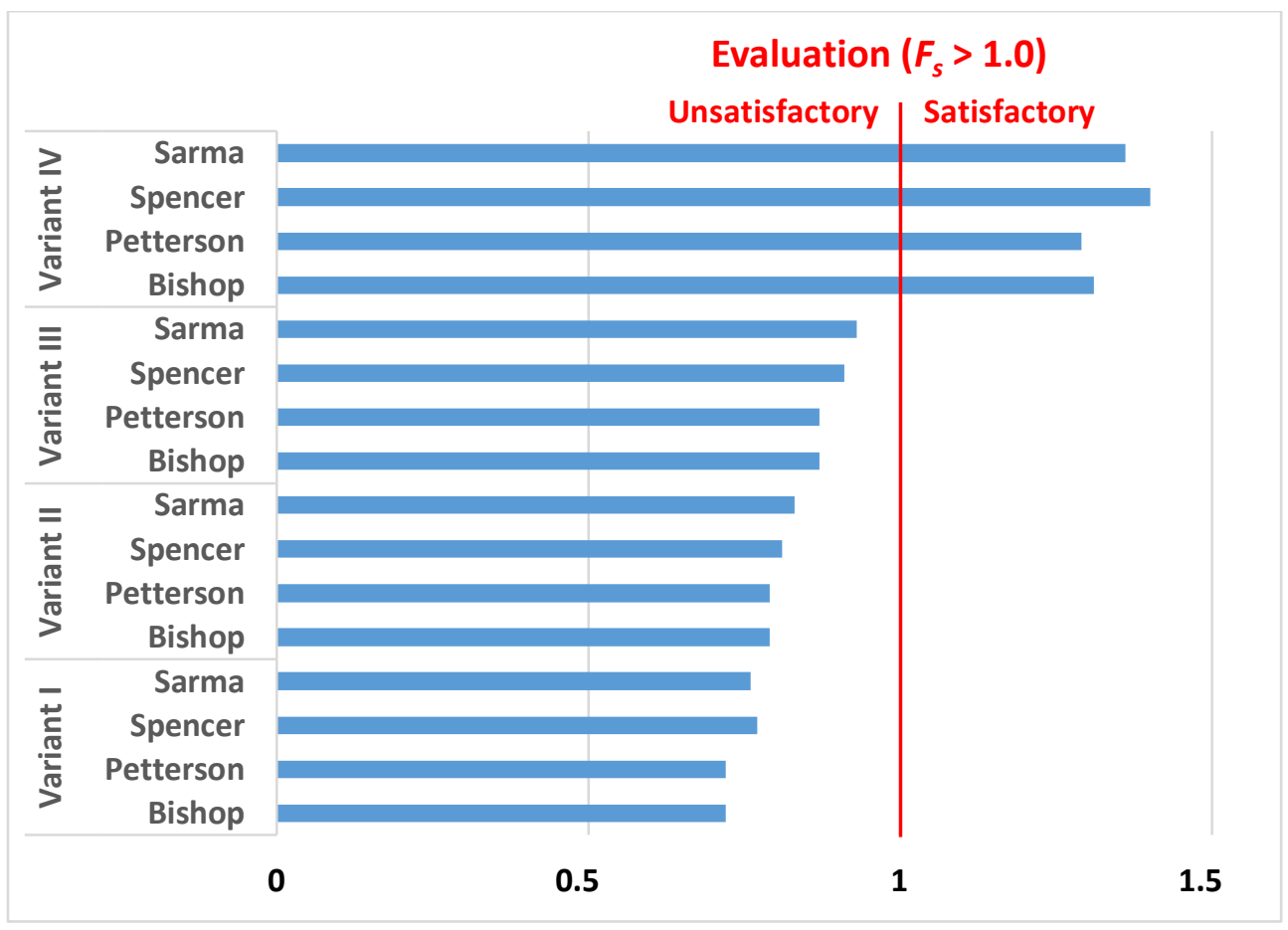

Fig. 2. Calculated factors of safety for Variant I to Variant IV. 
The stability analysis takes into account two basic principles. The first is an assumption that the slip surface developed will be circular (Petterson and Bishop Methods) and the second principle is that the slip surface will be polygonal (Sarma and Spencer Method).

\section{0}

1.0

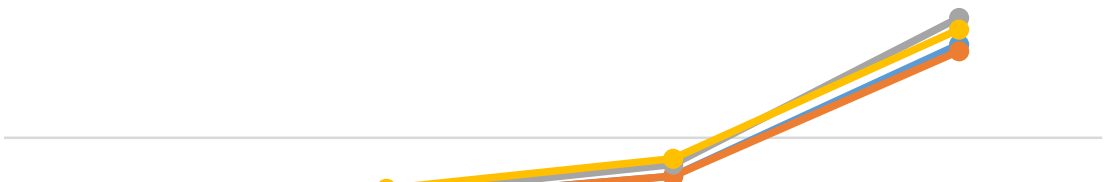

0.0

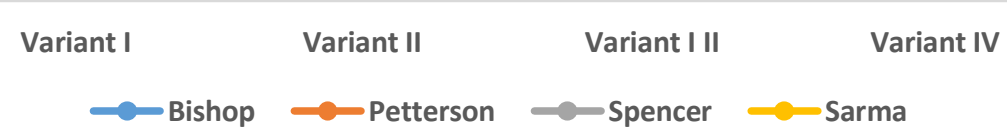

Fig. 3. Comparison of methods for the calculation of slope stability (Variant I to Variant IV).

Fig. 3 shows relatively good agreement of the calculated factor of stability methods, which take into account circular shear surface, as well as the methods that take into account shear polygonal surface. The difference in values in the calculated degrees of stability using these methods is only 0.07 .

\section{Conclusion}

Assessment of slope stability is performed for a safe, economical and useful design of slope for both natural and man-made slopes such as embankments, road cuts and excavations.

The stability of the slope can be assessed by the factor of safety that is a very useful index to be used when finding out how close or far is the slope from failure. A factor of safety may by calculate according to generated critical sliding surfaces which were found by using different methods (Bishop, Petterson, Sarma, Spencer).

Designed variants shows relatively good agreement of the calculated factor of stability methods, which take into account circular shear surface, as well as the methods that take into account shear polygonal surface.

Calculation of slope stability of the cut walls was realized in four variants, in which we analyzed possibilities to solve and improve the stability slope. Variant IV is satisfactory, but it occupies a large area and that means a large volume of earthwork. For further modelling of ensuring of slope stability, we want designed securing the cutting walls with earth nails and anchors. Reinforced of slope with nails and ground anchors can be used to improve the stability of a slope. Slope stability calculation is required to determine the safety factor of a slope [7]. The calculation of slope stability requires the layer description, 
the soil properties, such as soil cohesion, the internal friction angle, and the critical sliding surfaces.

This work was supported by the Scientific Grant Agency of the Ministry of Education of Slovak Republic and the Slovak Academy of Sciences under Project VEGA 1/0374/19.

\section{References}

1. E. Panulinova, S. Harabinova, Advanced Material Research, 969, 245-248 (2014)

2. Y. Sutejo, N. Gofar, Procedia Engineering, 125, 331-337 (2015)

3. P. Dzugas, Impact of Shear Strength on Slope Stability (Bachelor work, 62, 2018)

4. D. H. Cornforth, Landslides in Practice, Investigation, Analysis, and Remedial Preventive Options in Soils (John Wiley \& Sons, 2005)

5. G. Gianpaolo, Rock Slope Stability Analysis (A.A. Balkema Publishers,1994)

6. GEO 5 program (C) Fine Ltd.

7. P. B. Santoso, Yanto, A. Apriyono, R. Suryani, MATEC Web Conf., 195, 10 (2018) 